\title{
Flexicurité et marchés transitionnels du travail : esquisse d'une réflexion normative
}

Flexicurity and Transitional Labour Markets in a public policy Perspective

\section{Bernard Gazier}

\section{(2) OpenEdition}

1 Journals

Édition électronique

URL : http://journals.openedition.org/travailemploi/2340

DOI : 10.4000/travailemploi.2340

ISSN : 1775-416X

Éditeur

DARES - Ministère du Travail

Édition imprimée

Date de publication : 17 mars 2008

Pagination : 117-128

ISSN : 0224-4365

\section{Référence électronique}

Bernard Gazier, « Flexicurité et marchés transitionnels du travail : esquisse d'une réflexion normative », Travail et Emploi [En ligne], 113 | janvier-avril 2008, mis en ligne le 17 février 2011, consulté le 19 avril 2019. URL : http://journals.openedition.org/travailemploi/2340 ; DOI : 10.4000/travailemploi.2340 


\title{
Flexicurité et marchés transitionnels du travail: esquisse d'une réflexion normative
}

\author{
Bernard Gazier(*)
}

\begin{abstract}
L'auteur constate l'ambivalence du concept de flexicurité et l'examine à partir de la théorie des marchés du travail transitionnels (MTT). Les marchés transitionnels veulent être un complément ou un substitut aux marchés internes défaillants. Ils visent à procurer une mobilité protégée en agissant sur les transitions, considérées comme des passerelles appelées à devenir des objets de négociation et de gestion systématique; l'article présente la variété des outils susceptibles d'être combinés dans ce cadre, et adaptés à une logique de flexicurité conçue sur le long terme et sur une base négociée collectivement. A partir d'un programme de recherche européen, il fait le point sur les adaptations nationales du concept de flexicurité appliquées dans différents États membres.
\end{abstract}

L'idée de «flexicurité» est de plus en plus diffusée comme objectif de politique économique et sociale en Europe et dans le monde. Depuis 2005, l'Union européenne en a fait un des axes majeurs de ses préconisations, notamment dans le cadre de la Stratégie européenne de l'emploi (Commission EUROPÉENNE, 2006). Toutefois elle demeure controversée, tant dans sa définition que dans ses implications, voire son opportunité. L'enjeu est évidemment l'adaptation des marchés du travail aux défis des délocalisations, du chômage et de la précarité, par des transformations des conditions de leur fonctionnement. Il s'agit de reconfigurer la protection de l'emploi, les politiques de l'emploi et les politiques sociales afin, en liaison avec d'autres politiques dans les champs de la macroéconomie et de l'innovation, d'affronter la nouvelle donne de l'économie mondiale.

L'argument le plus souvent avancé est que, dans certains pays européens et notamment en France, la protection de l'emploi serait excessive, voire dissuasive, et qu'il conviendrait de la relâcher en compensant éventuellement cet affaiblissement par d'autres garanties données aux travailleurs, renforçant la protection sociale et les politiques actives de l'emploi. De grandes institutions internationales telles que le FMI et l'OCDE ont largement diffusé ce type de propositions (FMI, 2003; OCDE, 2006). Comme on l'a vu en France avec l'échec du contrat première embauche en 2006, cette perspective ne va pas sans résistances de la part des intéressés et des organisations syndicales.

(*) Matisse, université Paris 1, Bernard.Gazier@univ-paris1.fr
D'un autre côté, nous assistons à l'émergence d'une série de nouveaux droits et techniques de gestion et sécurisation des carrières: droits à recyclage et reconversion, à divers congés, parentaux, sabbatiques, de formation, droit au suivi individuel voire au coaching et aux bilans de compétences, à la validation des acquis de l'expérience, etc. Ces nouveaux droits ne font guère l'objet de controverses, et sont aisément perçus comme des avancées sociales. Il y a, de prime abord, deux faces de la «flexicurité », l'une associée à des menaces de déstabilisation, et l'autre porteuse de promesses.

Dans cet article, nous tentons, sous un angle normatif, de clarifier l'usage qui peut être fait du terme de «flexicurité», en adoptant le point de vue d'un programme de recherche et de propositions développé depuis une bonne dizaine d'années en Europe, celui des marchés transitionnels du travail.

Nous procéderons en deux temps. La première section propose un bref bilan des acquis et des développements et interrogations récents de ce programme de recherche. La seconde section interroge, dans la perspective ainsi tracée, l'idée de «flexicurité» dans ses dimensions, sa cohérence et ses usages possibles.

\section{Les marchés transitionnels du travail: une approche globale et dynamique du travail et de l'emploi}

Issus d'une réflexion pragmatique menée au début des années 1990 par des économistes du WissenschaftZentrum Berlin (WZB) (Schmid, 
1995a; ScHMID, 1995b), cette dernière référence constituant, dans les colonnes de Travail et Emploi, la première présentation en français de cette perspective; (Auer, Schmid, 1997), les marchés transitionnels du travail (désormais MTT) proposent une perspective de reconceptualisation et de réforme d'ensemble des marchés du travail européens pour les adapter aux défis et besoins du début du $\mathrm{XXI}^{\mathrm{e}}$ siècle.

\section{Les formulations initiales}

Les MTT consistent en l'aménagement systématique et négocié de l'ensemble des positions d'activité au sens large, traditionnellement considérées comme les marges de l'emploi, et qui deviennent ici les «transitions" : périodes de formation ou de congé parental, mi-temps tout court ou combiné à un autre mi-temps, associatif par exemple, préretraite à temps partiel... Ces périodes et ces occupations ont pour trait commun d'associer des activités jugées socialement utiles à une garantie temporaire de rémunération (qui peut combiner des financeurs divers), et de constituer des passerelles vers d'autres positions sur le marché du travail (SCHMID, GAZIER (dir.), 2002; GAZIER, 2005).

L'idée est ainsi que des transitions et des «passerelles » (1) sont devenues inévitables sur les marchés du travail actuels, qui doivent de toute manière fonctionner avec des travailleurs à réadapter sans cesse. Les itinéraires deviennent discontinus, ne serait-ce qu'à cause des obligations de recyclage. Les MTT organisent la recherche de nouveaux espaces et de nouvelles modalités de confrontations de l'offre et de la demande, à propos de nouveaux objets de négociation: les positions temporaires d'activité. La démarche est d'abord positive pour ensuite affirmer son ambition normative. Il s'agit d'identifier les «transitions» sur le marché du travail et autour de celui-ci, afin de repérer celles qui sont favorables à l'initiative et aux revenus des travailleurs (les «bonnes» transitions), pour les promouvoir et décourager les «mauvaises», celles qui conduisent à l'exclusion ou l'appauvrissement.

Comme l'avait proposé G. Schmid dès 1995, on peut identifier cinq champs principaux de transitions. Ils correspondent tout d'abord aux trois séquences traditionnelles de toute vie professionnelle: la formation (initiale ou continue), l'exercice d'une activité rémunérée (salariée ou non, à temps complet ou temps partiel...), et la retraite (progressive ou totale) et l'inactivité. Les deux domaines de mobilités additionnelles sont le chômage et les activités sociales utiles non rémunérées (tâches domestiques et familiales, bénévolat, militantisme).

(1) Le terme allemand à l'origine de la dénomination «transitionnel» est «übergange», soit «passerelles».
L'idée clé est l'existence, au sein de chacun de ces champs, de «transitions critiques» qui ont lieu au cours de la vie des travailleurs et qui sont susceptibles d'infléchir leur itinéraire en un sens socialement non souhaitable. Ces «transitions critiques» peuvent fort bien avoir une origine extérieure au marché du travail, et avoir été causées par un événement de nature privée: une naissance, un divorce, un parent en situation de dépendance... Mais elles peuvent aussi relever directement du marché du travail, comme dans le cas d'un licenciement ou d'une mutation. Les conséquences d'une mauvaise gestion ou d'une mauvaise protection peuvent être un appauvrissement temporaire ou durable, mais aussi un découragement personnel ou même une exclusion objective (cas, par exemple, des bassins du travail sinistrés).

Quatre principes permettent alors de définir les «bonnes transitions».

- Accroître la liberté individuelle (ou l'autonomie), en donnant aux personnes plus de pouvoir, non seulement en termes financiers via des transferts mais aussi en termes de participation aux décisions d'emploi qui les concernent. En échange de quoi les participants au marché du travail pourront accepter d'assumer plus de risques, plus de devoirs et d'obligations.

- Promouvoir la solidarité dans la gestion des risques sociaux et des risques associés au marché du travail. Ceci implique l'inclusion, dans les programmes de redistribution, des travailleurs les plus favorisés, qui courent moins de risques ou sont mieux à même de les assurer.

- Rechercher l'efficacité des mesures accompagnant les transitions, à travers un processus de spécialisation, coordination et coopération. Celuici prend le plus souvent la forme d'un mélange de contributions publiques et privées dans la formulation et la mise en œuvre des politiques correspondantes, et suppose des modalités négociées de prise de décision.

- Mobiliser l'arsenal des techniques de gestion des risques: contrôle, évaluation et autorégulation, par le biais d'une démarche largement décentralisée ou par le management par objectifs. Ce principe requiert la recherche d'une meilleure codétermination des acteurs concernés, que ce soit au niveau de la firme, au niveau local ou au niveau régional.

Les MTT sont facilités par l'arrivée de nouveaux acteurs et financeurs dans les négociations autour de l'emploi et des transitions. Par exemple, organiser des préretraites à temps partiel(2) peut se faire avec l'or-

(2) La préretraite à plein temps n'est pas une mesure obéissant à la logique des MTT, sauf dans certains cas «sociaux». En effet, elle est le plus souvent, quel que soit l'engouement dont elle bénéficie, irréversible, brutale, contraignante, unilatérale et coûteuse. 
ganisation de compléments d'emploi du temps au sein d'associations qui n'ont pas les moyens de financer un emploi à temps plein et peuvent accueillir des collaborateurs à temps partiel - cet emploi pouvant $\mathrm{du}$ reste être cofinancé par un partenaire municipal ou régional. Un tel dispositif a l'intérêt de compléter les revenus et l'activité des travailleurs vieillissants, en satisfaisant des besoins sociaux et sans alourdir la charge des finances publiques.

Au-delà de mesures spécifiques, la démarche des MTT est une démarche d'ensemble obéissant au principe «ma mobilité dépend de la vôtre». Il en résulte l'insistance mise sur des négociations locales associant de multiples acteurs susceptibles d'être cofinanceurs. Une série de questions de mise en œuvre surgit alors, portant notamment sur le coût et la cohérence de ces arrangements locaux (GAZIER, 2000; GAZIER, 2002). Les MTT consistent d'abord en réaménagement des mesures existantes, et prennent appui sur les avantages des cofinancements y compris en provenance du travailleur bénéficiaire de la «transition», situations dans lesquelles chaque participant a intérêt au succès de la «transition». Plus que des dépenses nouvelles, il s'agit plutôt de la réappropriation par les acteurs locaux des sommes souvent très importantes consacrées par l'État aux politiques de l'emploi, et de la tentative de réduire les externalités négatives du marché du travail pour capter les externalités positives. On peut donc établir des conditions de maîtrise des coûts. La question de la cohérence apparaît par exemple lorsque l'on envisage des régions riches proposant un ensemble sophistiqué de «transitions», pendant que des régions pauvres en sont réduites à une offre minimale. Le moyen de combattre cette incohérence est une politique de transferts, qui suppose d'élaborer et d'utiliser des critères de besoins locaux en termes de «transitions».

En somme, cette gestion systématique et négociée des «transitions» revient à accorder de nouveaux droits aux travailleurs, qu'ils soient intégrés dans des «marchés internes» leur apportant déjà de multiples garanties de carrière, ou qu'ils soient chômeurs ou cantonnés aux "marchés secondaires» et disposant d'emplois avec peu de perspectives d'avancement et de stabilisation. Nous étions partis d'une perspective de réforme des politiques de l'emploi, voici désormais que se dessinent des propositions de réforme de la relation salariale au sein des firmes, en faveur des stakeholders et au détriment des actionnaires. Les MTT visent à réduire la distance entre les plus protégés des travailleurs et les autres, mais en apportant plus de droits à tous. Ce qui a pour effet de bénéficier aux moins favorisés, les mobilités des un(e)s permettant celles des autres. Cette conception généralisée et dynamique de l'activité et des trajectoires permet enfin de réintégrer directement l'informel de la division du travail dans l'espace de la négociation et de la régulation sociale et se relie directement aux travaux menés en termes de droits de tirage sociaux et de statut d'activité (SuPIOT, 1999).

\section{Quelques interrogations et avancées récentes}

Les questions et mises en cause ont été relativement nombreuses. Les principales relèvent de trois ordres. Tout d'abord, une série d'auteurs ont estimé que l'angle d'attaque et d'intervention retenu, les «transitions», pouvait entretenir une négligence des conditions de pérennité de l'emploi, et donc orienter l'action publique vers une mise en mouvement excessive et non désirée des travailleurs, ceuxci restant fondamentalement en quête de stabilité (Ramaux, 2006). Cette critique a ceci de piquant qu'elle s'appuie souvent sur les travaux qui mettent en évidence le maintien, voire le renforcement, actuel de relations de travail et d'emploi à long terme, parmi lesquels se détachent ceux de Peter Auer, l'un des inspirateurs initiaux des MTT (AuER, CAZES, 2003). Une variante s'inquiète des effets sur les inégalités de "genre», en soulignant que les femmes effectuant davantage de "transitions» que les hommes, elles risquaient de devenir de plus en plus, à leur détriment, la variable d'ajustement (JEPSEN, 2005).

Une seconde série de considérations voit dans la démarche un simple processus de redistribution de l'emploi (DE KonING, 2002) assimilable à d'autres démarches de partage du travail et se heurtant aux mêmes limites (coûts de transaction et de coordination, effets désincitatifs pour les entreprises comme pour les personnes).

Un troisième groupe de critiques consiste à se saisir du terme "marché» dans les MTT pour estimer qu'ils consistent à rabattre sur le marché, même «équipé», des formes de coordination non marchandes (EYMARD-DUVERNAY, 2006).

L'essentiel des réponses a consisté à remarquer qu'agir sur les mobilités et les transitions apparait comme une nécessité, voire une opportunité, dans un contexte d'érosion et de transformation des «marchés internes du travail». Les MTT, processus négocié et collectif d'attribution de positions et de rémunérations, n'impliquent aucun diagnostic en termes d'accroissement des mobilités sur le marché $\mathrm{du}$ travail, et encore moins de prescription en ce sens. Ils se veulent un complément, voire un substitut, aux «marchés internes» défaillants et visent à apporter ce que l'on peut appeler une «mobilité protégée».

Au-delà de ces interrogations et réponses, il est possible de noter une double évolution. D'une part, la mise en œuvre des démarches «transitionnelles», initialement envisagée dans un cadre systémique à vocation globalisante, s'est enrichie d'un fondement plus microéconomique en termes de «gestion du risque social». Prenant appui sur les travaux de psychologie économique consacrés à la perception et à la gestion du risque, G. Schmid (SchMID, 2006) a introduit l'idée de social risk management, géné- 
ralisant une perspective assurantielle et préventive à une série de risques sociaux nouveaux ou renouvelés. Il en va ainsi notamment des situations affrontées par de jeunes travailleurs et travailleuses en début de carrière, devant tout à la fois trouver un partenaire, un logement, un emploi, une orientation professionnelle et les moyens d'élever de petits enfants. Ou encore des pressions renforcées vécues par les salariés à qui l'on demande de plus en plus d'initiatives dans des contextes de moins en moins prévisibles.

D'autre part, la réflexion sur le concept même de «transition» et de carrière s'est poursuivie. L'insistance est désormais mise sur les processus tantôt réversibles et tantôt irréversibles qui structurent les trajectoires dans les «cycles de vie» (ANXo, Erhel, 2006; Erhel, 2007). L'idée de «noyaux de stabilité» (AUER, GAZIER, 2006), tire les conséquences de l'idée de bon sens que les «transitions», sauf à désigner toute séquence de carrière, doivent logiquement mener quelque part. Les négociations doivent alors porter aussi sur l'aménagement de positions productives autour de l'entreprise, s'appuyant par exemple sur les métiers, les branches, les territoires, les fonctions publiques nationales et locales, et l'économie non marchande. On retrouve alors l'échelon territorial, qui semble le plus adapté pour établir simultanément des «noyaux de stabilité» et des «transitions» y conduisant.

\section{La flexicurité vue des marchés transitionnels}

À propos de la «flexicurité», les MTT suggèrent des réponses à trois séries de préoccupations. $\mathrm{La}$ première porte sur la nécessité de prendre en compte sinon la totalité du moins l'essentiel des dimensions pertinentes. Il en résulte une opposition simple entre deux versions au moins de la «flexicurité», l'une se révélant tronquée et devant être écartée. On doit ensuite s'intéresser à la variété des outils qui peuvent être mobilisés dans ce cadre, et à la manière dont ils peuvent être combinés. Enfin, il convient dans le cadre européen, de prendre en compte la diversité des situations nationales et «sociétales».

\section{Distinguer deux «flexicurités "}

La vue la plus répandue et la plus conventionnelle de la «flexicurité» procède d'une double réduction. D'une part, il s'agit de se focaliser sur une dualité asymétrique de besoins dans le contexte économique et social actuel, les firmes demandant plus de flexibilité et les travailleurs plus de sécurité. On laisse donc de côté les besoins symétriques pourtant évidents, les firmes ayant besoin de sécurité, notamment quant à la disponibilité d'une main-d'œuvre adéquate, et les travailleurs demandant davantage de flexibilité, par exemple pour mieux concilier leur vie professionnelle et familiale, ou encore pour évoluer dans leur carrière.
D'autre part, le principe le plus couramment avancé consiste en un échange auquel consentiraient les travailleurs, entre une protection de l'emploi amoindrie et davantage de protection sociale assortie d'une prise en charge renforcée par les politiques actives de l'emploi. On arrive alors à la représentation bien connue du «triangle d'or» danois ( $c f$. figure 1 ci-dessous). Deux circuits rapides sont alors créés pour les travailleurs. L'un consiste à passer rapidement de l'emploi au chômage et du chômage à l'emploi en profitant d'une indemnisation généreuse, une protection de l'emploi relativement faible permettant au marché du travail d'avoir un haut niveau de séparations mais aussi d'embauches. L'autre circuit passe par les «politiques actives de l'emploi», qui prennent le relais lorsque le temps passé au chômage risque de se prolonger.

L'essentiel de cette vision de la «flexicurité» se trouve ainsi dans la création d'un marché du travail high speed, combinant un faible niveau de réglementation avec un fort niveau de socialisation et de prise en charge. Au-delà des «faits stylisés» caractéristiques de l'expérience danoise: forte mobilité des travailleurs entre les emplois, très bonne indemnisation du chômage notamment pour les bas salaires, et poids important des interventions des politiques «actives» de l'emploi, on retrouve alors les préconisations d'amoindrissement de la législation protectrice de l'emploi. En France le rapport Cahuc-Kramarz constitue un exemple très élaboré de cette démarche (CAhuc-Kramarz, 2005).

Figure 1

Le «triangle d'or» danois

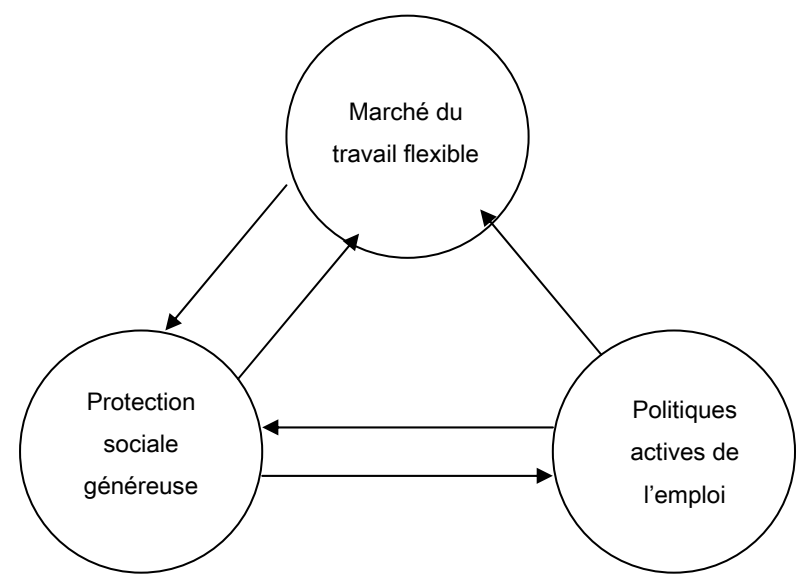

Il a toutefois souvent été observé que ce schéma repose, au moins dans le cas danois, sur plusieurs particularités qui en conditionnent le fonctionnement. Deux valent d'être mentionnées ici (BARBIER, 2006; Boyer, 2006). Petit pays dont l'économie est essentiellement formée de PME, le Danemark a conclu très anciennement avec ses syndicats extrêmement puissants une série d'accords compensant une grande facilité à embaucher et licencier par une implication très forte des syndicats dans la vie des entreprises et le contrôle du marché du 
travail. Ensuite, la main-d'œuvre danoise bénéficie d'un haut niveau d'ensemble de qualification, et de politiques de formation professionnelle et continue très développées. Le type d'ajustement qui a été recherché et obtenu repose ainsi sur une configuration sociétale dont certains éléments clés sont oblitérés par le schéma du «triangle d'or».

À ce point, les représentations en termes de «marchés transitionnels» permettent de proposer une extension de ce même schéma, dans deux directions au moins. L'une part des exigences et réalisations en termes de formation, et conduit à souligner le rôle potentiel des firmes elles-mêmes dans la production et l'entretien de compétences. Qu'il s'agisse des politiques de formation menées par les firmes elles-mêmes ou des partenariats et réseaux qu'elles peuvent nouer, par exemple dans les filières d'apprentissage, on a là un premier pôle d'ajustement négligé dans le schéma précédent. On peut ensuite introduire le territoire comme second pôle d'ajustement, en entendant par là la capacité des acteurs locaux à générer des positions d'emploi et d'activité, par exemple par la constitution de groupements d'employeurs ou l'aménagement séquentiel d'emplois saisonniers. Cette double introduction enrichit l'analyse du fonctionnement du marché du travail. Elle prend son sens opérationnel dans le cas où des ajustements négociés supplémentaires, en termes de construction de compétences et en termes de trajectoires locales, font l'objet d'interventions politiques explicites et ne sont pas laissés au bon vouloir des acteurs concernés.

Le schéma précédent est alors conservé mais transformé et, de «triangle», devient en quelque sorte un «carré» dont une présentation possible est donnée par la figure 2, ci-dessous.

Figure 2

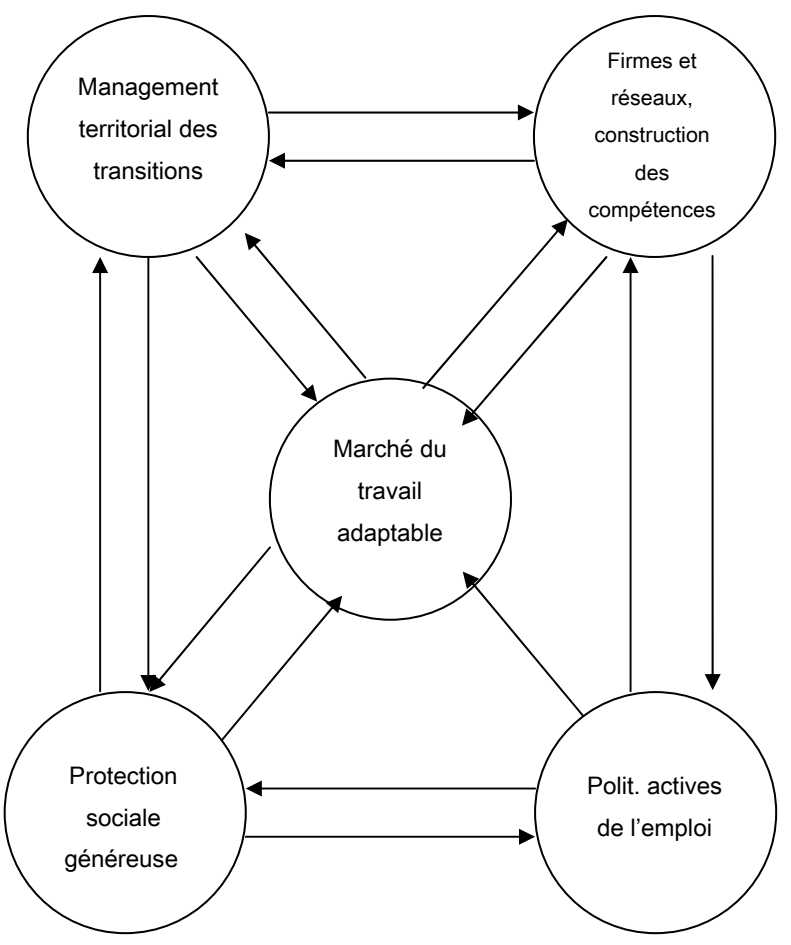

Une seconde conception de la «flexicurité» apparaît alors, qui s'organise autour d'un marché du travail «adaptable» au sens où il ne s'agit plus simplement de la facilité avec laquelle les entreprises peuvent embaucher ou licencier. Le lecteur familier des textes élaborant les «marchés transitionnels » remarquera aisément la grande proximité entre ce schéma et les présentations initiales des MTT (Schmid, 1995a et b) en termes de «champs de transitions ».

Il en résulte que la première version de la «flexicurité» a toutes les chances d'être mystifiante en se limitant à une gamme trop étroite d'ajustements et doit donc être rejetée. Les canaux qu'elle décrit sont trop partiels pour être opérationnels, sauf à croire dans les effets a priori bénéfiques d'une dérégulation du marché, éventuellement compensée par ailleurs. Une des caractéristiques essentielles des propositions faites par CAHUC et KRAMARZ (op. cit., 2005) est d'organiser le désengagement complet des entreprises en ce qui concerne les carrières de leurs salariés, hors du paiement d'une taxe sur les licenciements. Cette démarche tronque ainsi l'analyse et néglige les apports d'autres sources de coordination et d'adaptation. Parmi elles, l'ensemble des démarches par lesquelles les entreprises construisent, en interne et le plus souvent en partenariat avec des organismes de formation, des compétences transférables, et garantissent un niveau d'employabilité tel que leurs salariés peuvent retrouver des emplois sans subir de déclassement salarial en cas de perte d'emploi. L'offre locale des mobilités et transitions est le second domaine négligé, implicitement réduit aux conditions d'ajustements de l'offre et de la demande d'emploi, et n'ouvrant ni sur les conditions de conciliation de la vie privée et de la vie professionnelle, ni sur l'aménagement des trajectoires en liaison avec les collectivités locales et le réseau des organisations privées non marchandes. Un débat sur la définition et la possible redéfinition des responsabilités des entreprises - et des appuis qu'elles peuvent recevoir - dans ces deux domaines clés est ainsi occulté et réduit à une alternative simpliste entre engagement supposé trop contraignant et désengagement total (GAZIER, 2007).

\section{Construire les conditions d'une dynamique de "flexicurité"}

Quiconque cherche à rentrer dans le détail des pratiques et outils censés générer la «flexicurité» ne peut qu'être frappé par la variété, voire l'hétérogénéité, des dispositifs et des moyens susceptibles d'être mobilisés. Parmi les efforts d'élaboration et de mise en ordre, se détachent ceux du sociologue hollandais Ton Wilthagen (Wilthagen, Tros, 2004; Wilthagen, 2005). Ces apports ont été condensés dans un tableau qu'il sera commode d'appeler la «matrice de Wilthagen», dont une version récente reprise par la Fondation de Dublin est donnée cidessous ( $c f$. tableau 1). 
Tableau 1

La «matrice de Wilthagen» $(1)$

\begin{tabular}{|l|l|l|l|l|}
\hline Flexibilité & Sécurité de l'emploi occupé & \multicolumn{1}{|c|}{ Sécurité de l'emploi } & \multicolumn{1}{c|}{ Sécurité du revenu } & \multicolumn{1}{c|}{ Sécurité combinée } \\
\hline $\begin{array}{l}\text { Flexibilité externe } \\
\text { numérique }\end{array}$ & $\begin{array}{l}\text { Types de contrat de travail } \\
\text { Législation protectrice de } \\
\text { l'emploi } \\
\text { Retraite anticipée }\end{array}$ & $\begin{array}{l}\text { Services de l'emploi } \\
\text { / politiques actives de } \\
\text { l'emploi } \\
\text { Formation / formation } \\
\text { tout au long de la vie }\end{array}$ & $\begin{array}{l}\text { Indemnisation du } \\
\text { chômage. } \\
\text { Autres prestations } \\
\text { sociales } \\
\text { Salaire minimum }\end{array}$ & $\begin{array}{l}\text { Protection contre le } \\
\text { licenciement durant } \\
\text { divers dispositifs de } \\
\text { congés }\end{array}$ \\
\hline $\begin{array}{l}\text { Flexibilité interne } \\
\text { numérique }\end{array}$ & $\begin{array}{l}\text { Chômage partiel / horaires } \\
\text { courts / temps partiels }\end{array}$ & $\begin{array}{l}\text { Législation protectrice } \\
\text { de l'emploi } \\
\text { Formation / formation } \\
\text { tout au long de la vie }\end{array}$ & $\begin{array}{l}\text { Allocation supplémen- } \\
\text { taire compensant un } \\
\text { temps partiel } \\
\text { Bourses d'études } \\
\text { Allocations maladie }\end{array}$ & $\begin{array}{l}\text { Différents types de } \\
\text { congés } \\
\text { Retraite à temps partiel }\end{array}$ \\
\hline $\begin{array}{l}\text { Flexibilité } \\
\text { fonctionnelle }\end{array}$ & $\begin{array}{l}\text { Enrichissement du travail } \\
\text { Formation } \\
\text { Prêt de travailleurs } \\
\text { Sous-traitance } \\
\text { Externalisation }\end{array}$ & $\begin{array}{l}\text { Formation / formation } \\
\text { tout au long de la vie } \\
\text { Rotation des emplois } \\
\text { Travail en équipes } \\
\text { Polyvalence }\end{array}$ & $\begin{array}{l}\text { Systèmes de rému- } \\
\text { nération reliés à la } \\
\text { performance }\end{array}$ & $\begin{array}{l}\text { Modulations volon- } \\
\text { taires du temps de } \\
\text { travail }\end{array}$ \\
\hline $\begin{array}{l}\text { Flexibilité du } \\
\text { coût du travail/du } \\
\text { salaire }\end{array}$ & $\begin{array}{l}\text { Ajustements locaux des coûts } \\
\text { du travail } \\
\text { Modulation/réduction des paie- } \\
\text { ments de sécurité sociale }\end{array}$ & $\begin{array}{l}\text { Modulation/réduc- } \\
\text { tion des paiements de } \\
\text { sécurité sociale } \\
\text { Subventions à l'emploi } \\
\text { Primes pour l'emploi }\end{array}$ & $\begin{array}{l}\text { Accords collectifs sur les } \\
\text { salaires } \\
\text { Allocation compen- } \\
\text { satoire pour horaires } \\
\text { réduits }\end{array}$ & $\begin{array}{l}\text { Modulations volon- } \\
\text { taires du temps de } \\
\text { travail }\end{array}$ \\
\hline
\end{tabular}

(1) European Commission for the Improvement of Living and Working conditions, 2007, p. 4.

Cette grille repose sur une série de distinctions élémentaires, tant en ce qui concerne la flexibilité dont les firmes pourraient avoir besoin que la sécurité demandée par les travailleurs. Dans cette présentation, la flexibilité est ou bien numérique (interne ou externe), ou bien non numérique: soit fonctionnelle (ce terme rassemblant ici les possibilités d'ajustements qualitatifs internes tels que la formation ou la polyvalence, mais aussi les choix de flexibilité organisationnelle externe tels que le recours à la sous-traitance), soit en termes de coûts du travail modulés ou réduits. La sécurité, quant à elle, oppose la sécurité de l'emploi occupé (ce que l'anglais désigne par job security) et la sécurité de l'emploi ou de l'employabilité, qui se centre sur la capacité à obtenir rapidement un emploi acceptable ou à en changer (ce que l'anglais désigne par employment security). Viennent ensuite la sécurité de revenu en cas de perte d'emploi, et la sécurité résultant de la capacité à combiner diverses allocations et positions d'activité ou d'inactivité (combination security).

Comme on le voit, il résulte de cette grille la mise en évidence et la classification d'un vaste ensemble de mécanismes et d'outils variés, dans une perspective qu'il est aisé de rapprocher de la philosophie générale des MTT. Les «transitions » sont ici placées en complément des adaptations pouvant affecter l'emploi occupé et viennent démultiplier l'espace des arrangements. En particulier, l'insistance mise sur la sécurité de l'emploi ou de l'employabilité se double d'une réflexion sur des garanties de revenu, cependant que la «sécurité combinée» retrouve l'intuition de départ des MTT.

Toutefois, ces apports ne vont pas sans d'importantes limites qui apparaissent lorsque cette matrice est utilisée à des fins autres qu'un simple repérage des outils possibles, par exemple pour tenter d'établir un «menu national» en fonction de la situation et des acquis antérieurement développés. En effet, au-delà de critiques portant sur les rubriques retenues, le risque est de tomber dans un fonctionnalisme postulant la légitimité de l'usage conjoint d'une palette de moyens.

En ce qui concerne d'abord les rubriques, on peut s'étonner que la flexibilité fonctionnelle ne donne pas lieu à distinction entre une flexibilité fonctionnelle interne et externe à l'entreprise. Polyvalence et recours à la sous-traitance, formation en interne et en externe pourraient ainsi être distingués. Ensuite et surtout, le rôle des ajustements en termes de coûts du travail n'est pas clair dans cette clas- 
Tableau 2

La «matrice Schmid»(1)

\begin{tabular}{|c|c|c|c|c|}
\hline Flexibilité $\quad$ Sécurité & Sécurité du poste & Sécurité de l'emploi & Sécurité du revenu & Sécurité d'option \\
\hline Externe numérique & $\mathrm{d}$ & $\mathrm{d} / \mathrm{c} / \mathrm{v}$ & $\mathrm{d} / \mathrm{c} / \mathrm{v}$ & $\mathrm{d}$ \\
\hline Interne numérique & $\mathrm{c}$ & $\mathrm{c}$ & $(\mathrm{d}) / \mathrm{c}$ & $\mathrm{d} / \mathrm{c}$ \\
\hline Interne fonctionnelle & $\mathrm{c}$ & $\mathrm{c}$ & $\mathrm{d} / \mathrm{v}$ & $(d) / c$ \\
\hline Externe fonctionnelle & $\mathrm{c}$ & $\mathrm{d} / \mathrm{c} / \mathrm{v}$ & $\mathrm{d} / \mathrm{v}$ & $\mathrm{d} / \mathrm{c}$ \\
\hline
\end{tabular}

$\mathrm{d}=$ dilemme $\mathrm{c}=$ complémentarité $; \mathrm{v}=$ cercle vicieux ; les parenthèses dénotent des situations logiquement possibles mais peu probables.

(1) LeschKe, Schmid, Griga, 2006.

sification. D'une certaine manière, toute flexibilité a une traduction en termes de réductions de coûts, ce qui se devrait se voir en particulier pour les licenciements, qui ici relèvent (logiquement) de la rubrique «flexibilité externe numérique». Ensuite et de manière plus gênante, les rémunérations à la performance sont introduites dans la ligne «flexibilité fonctionnelle» et non pas la ligne «flexibilité du coût du travail et des salaires »; et elles impliquent une instabilité du revenu alors qu'elles relèvent (en colonne) de l'objectif de sécurité du revenu. La prise en compte à cette place de ces dispositifs vient ainsi mettre directement en crise la classification. On peut se demander du reste si la présence de ces ajustements de coûts et de salaires ne traduit pas une représentation implicite d'ajustements traditionnels de marché (par les prix et les coûts) venant s'offrir en alternative séparée.

Certes, ces limites peuvent trouver remède dans une reformulation plus cohérente des rubriques. Toutefois, et plus important, l'orientation générale de la grille suscite elle-même une réticence de fond. En effet, elle présente comme une boîte à outils dans laquelle il faudrait puiser pour adapter le «menu» de la «flexicurité» aux besoins et opportunités locaux. Or ce fonctionnalisme implicite n'est guère convaincant, et les travaux menés en termes de MTT offrent deux séries d'arguments pour s'en affranchir. Tout d'abord, la prise en compte des interactions entre l'ensemble des «transitions» exclut le simple rassemblement d'une liste d'outils dont on n'examinerait pas les conditions de fonctionnement conjoint dans un contexte donné. Ensuite, les travaux plus récents en termes de «gestion sociale du risque » (Schmid, 2006) mettent en évidence les bases individuelles et collectives de la construction sociale de la perception des risques. En particulier, les perceptions individuelles et de groupe varient selon le type de risque considéré. La visibilité des risques tout comme l'ampleur des menaces et des opportunités qui leur sont associées varient ainsi de manière cruciale selon le positionnement social et le contexte. Pour nous en tenir aux dispositifs déjà évoqués de rémunération variant avec la performance, on conçoit qu'ils n'aient pas le même sens, et surtout pas les mêmes conséquences en termes d'insécurisation ou de sécurisation, dans des entreprises où l'individualisation des tâches et de la mesure des performances est admise, et dans des contextes où l'accent demeure sur l'engagement du collectif de travail.

Il résulte de ces insuffisances et interrogations qu'une reformulation, plus cohérente et plus fidèle aux orientations «transitionnelles» est à l'ordre du jour. Elle a été proposée en 2006 par G. Schmid lui-même, dans un travail mené en collaboration (LeschKe et al., 2006), et on peut l'intituler la «matrice Schmid» (cf. tableau 2). La reformulation, en se focalisant d'emblée sur la résultante de chaque intersection de ligne et de colonne, fait toutefois évoluer le statut même de la matrice, passant d'un quadrillage d'outils à l'identification de complémentarités problématiques.

Cette seconde matrice reprend largement, en les rendant plus cohérentes, les subdivisions envisagées plus haut. L'intitulé «sécurité d'option» rend plus explicite l'ouverture des choix qui sont offerts aux travailleurs, au-delà de l'emploi traditionnel. La rubrique de réduction des coûts/salaires n'apparaît plus, son contenu étant ventilé dans les autres types de flexibilité. La distinction entre flexibilités fonctionnelles interne et externe aux entreprises est désormais mise en avant. Elles rassemblent tout ce qui ne relève pas d'ajustements numériques et mobilise les qualités et les prix. Dans un cas, nous trouvons notamment les capacités d'apprentissage sur le tas et de réorientation productive des travailleurs, ainsi que la capacité de flexibilité nominale des salaires, par exemple sous forme de primes. Dans l'autre, ce sont les formations en externe, la sous-traitance et la flexibilisation réelle des salaires (pour plus de détails quant au contenu de chaque case, on se reportera au travail de LeschKe et al., op. cit.).

Mais l'attention se déplace vers le type de cohérence que l'on peut attendre des croisements entre 
différents types de sécurité et de flexibilité. Les trois possibilités sont le dilemme (trade-off ici conçu comme un jeu à somme nulle) où il faut renoncer à de la sécurité pour obtenir davantage de flexibilité, ou inversement; la complémentarité, où l'accroissement de l'une permet l'accroissement de l'autre, et le cercle vicieux, complémentarité négative où les tentatives d'accroissement de l'une se font non seulement au détriment de l'autre, mais rejaillissent in fine sur la première et font baisser conjointement les deux.

Sans rentrer dans le détail des seize cases distinguées ( $c f$. LESCHKE et al., op. cit.), on observe pour la première colonne une situation aisée à décrire. La sécurité de l'emploi détenu, où l'on vise à protéger les travailleurs en place, est évidemment en relation de trade-off avec la flexibilité externe numérique (augmenter cette dernière, c'est abaisser d'autant le niveau de la première); en revanche les autres flexibilités, qui permettent de garder les travailleurs en place tout en faisant varier les conditions de leur utilisation, sont en relation de complémentarité. Toutefois, la matrice montre le type de difficultés et d'opportunités que l'on rencontre en envisageant d'autres types de sécurités. Dans la colonne "sécurité de l'emploi et de l'employabilité », deux possibilités de cercles vicieux apparaissent. Tenter de développer simultanément la sécurité de l'emploi et la flexibilité externe numérique et/ou fonctionnelle, c'est le pari dangereux de la «flexicurité» au sens tronqué abordé ci-dessus: développer des formes précaires d'emploi et d'externalisation, en espérant que la mise en mouvement du marché du travail permettra d'absorber les travailleurs ainsi brassés. Or une spirale de défiance et de faible motivation peut s'instaurer, avec une mise en cause de l'investissement réciproque des firmes dans leurs salariés et des salariés dans leurs firmes, processus qui pourrait conduire à une contraction de l'économie et donc à des pressions renforcées en faveur de l'externalisation et des emplois précaires. Il en va de même pour la sécurité de revenu, dont les sources de financement pourraient se contracter avec le recours croissant à l'externalisation. Spirales de défiance et crises de financement constituent ainsi les deux risques de cercles vicieux envisagés par cette matrice, qui sort ainsi du fonctionnalisme implicite pour envisager les conditions de fonctionnement conjoint d'une série d'outils et de coordinations.

Cette seconde matrice recèle un contenu qui peut sembler décevant au regard de la richesse instrumentale et apparemment opérationnelle de la précédente. Mais, précisément, elle montre que le contenu opérationnel n'était pas satisfaisant. Trois cases sur les seize montrent des situations où tout est possible, aussi bien le cercle vicieux que le dilemme ou le cercle vertueux. Cinq toutefois ne montrent que de la complémentarité; les risques de cercles vicieux sont eux aussi au nombre de cinq. Et elle opère un déplacement vers des stratégies d'évitement de ces derniers, au profit de la recherche de complémentarités. Elle ouvre ainsi un champ de recherche et de propositions dans la ligne des conceptions en termes de MTT évoquées plus haut, qu'il s'agisse du jeu interrelié des mobilités, ou du «management du risque social» tout au long du cycle de vie.

\section{Transitions et "flexicurité" en Europe: quelques jalons}

La place manque dans cette contribution pour aborder la variété des situations en Europe, ou pour examiner comment les politiques de développement de la «flexicurité» se relient aux autres politiques économiques et sociales tant nationales qu'européennes. On s'en tiendra ici à quelques remarques générales (pour plus de développements, voir par exemple ScHMID, 2008).

Tout d'abord, l'Europe se caractérise par une forte diversité de situations et contraintes nationales au regard de la «flexicurité». On pourrait ici reprendre la célèbre typologie d'Esping-Andersen et l'appliquer aux ajustements faits sur le marché du travail et autour de lui (GAZIER, LECHEVALIER, 2006). Les marges de manœuvre diffèrent ainsi fortement dans un régime de type anglo-saxon (qui privilégie les ajustements de flexibilité externe) ou de type nordique (qui part de l'adaptabilité d'une maind'œuvre homogène et bien formée). Mais le plus pertinent pour notre propos se situe à un niveau plus fin. Par exemple le niveau de protection de l'emploi diffère fortement entre le Danemark et la Suède. Ce dernier pays, ayant de grandes firmes fortement présentes sur son territoire, a fait le choix d'une protection élevée pour les travailleurs en poste, ce qui conduit à un équilibrage différent des sécurités et des flexibilités. On est alors conduit, pour certains mécanismes d'ajustements, à reprendre le concept d'«équivalent fonctionnel», qui montre que des résultats sinon identiques du moins largement équivalents peuvent être obtenus pas des processus et des institutions très différents.

Un autre point essentiel vient différencier les expériences «continentales». On sait que cette catégorie, intermédiaire selon Esping-Andersen, regroupe des pays ayant initialement fondé leur protection sociale sur les cotisations des travailleurs et non pas sur l'impôt. Selon la prévalence de l'intégration par des «marchés internes du travail» ou par des «marchés professionnels», la situation n'est toutefois pas la même en matière de sécurité et de flexibilité. Dans la logique de formation «duale» qui alimente les «marchés professionnels», des travailleurs aux qualifications transférables et bénéficiant d'un niveau relativement élevé de qualification peuvent affronter relativement facilement l'épreuve d'un changement d'entreprise et espérer des reclassements préservant au moins en partie le niveau de salaire antérieurement obtenu; tandis que les travailleurs intégrés avec peu de formation 
initiale dans des «marchés internes» (et c'est le cas en France) vont se trouver en quelque sorte piégés en interne. Leurs qualifications acquises sur le tas sont en effet largement spécifiques à leur entreprise, et donc peu ou pas transférables. Il en résulte l'anticipation de fortes difficultés de reclassement et la crainte de pertes massives de revenu en cas de changement d'entreprise, donc l'accent mis sur la protection de l'emploi détenu. Mais ces salariés ne sont que faiblement redéployables en interne, compte tenu de leur faible niveau de formation initiale générale. On observe ainsi dans cette configuration un cumul spécifique de pressions et de difficultés.

Face à cette variété de situations et de défis, la base commune de l'Europe ne doit pas être sousestimée. D'une part un assez bon niveau moyen de formation des travailleurs ouvre, en dépit des problèmes que nous venons d'identifier, une large palette d'adaptations possibles. D'autre part, le rôle souvent actif du dialogue social favorise des arrangements négociés et donc des relations de confiance entre les partenaires sociaux. De tels points d'appui constituent ainsi des atouts en Europe, notamment lorsqu'il s'agit d'éviter les cercles vicieux identifiés plus haut.

Si ceux-ci ont été présentés dans un cadre national, on doit souligner leur importante dimension européenne. Il en est ainsi de la gestion des restructurations et des délocalisations, dont une part significative est induite par la dynamique même de la construction européenne et notamment l'élargissement aux dix nouveaux pays membres admis en 2004. Ces pays affrontent des défis particulièrement aigus en matière de «flexicurité» avec l'apparition et le développement chez eux d'une flexibilité du travail et de l'emploi tout à fait excessive et contre-productive (CAZES, NESPOROVA, 2001; 2007). D'autre part, l'arrivée de firmes issues de l'Europe de l'Ouest, mettant à profit le faible niveau du coût du travail et la présence de nouveaux débouchés, met en évidence une responsabilité de l'Union ellemême en ce qui concerne la sécurité et le redéploiement des travailleurs de l'Ouest ayant perdu leur emploi de ce fait(3). Le socle de droits et de garanties, consistant dans les pays de l'ancienne Europe, fait donc l'objet de très fortes tensions.

Les débouchés de la "flexicurité» en Europe peuvent ainsi être esquissés tant au niveau national ou régional qu'au niveau de l'Union. Deux réponses doivent être distinguées. La première, a minima, consiste à plaider pour des «chemins» de réformes qui mobilisent de manière différenciée l'arsenal précédemment repéré, selon quelques grandes configurations de rigidités et d'insécurités établies

(3) Le «Fonds d'ajustement structurel» instauré en 2005 est un petit pas dans cette direction. Il ne devrait toutefois concerner que les travailleurs ayant perdu leur emploi du fait de délocalisations à l'extérieur de l'Union, et reste de dimension modeste. afin de baliser l'éventail des défis. C'est ce qui est fait dans le bref rapport Flexicurity Pathways remis en 2007 à la Commission européenne (WiLTHAGEN, 2007). Ce texte distingue quatre configurations qui, sans être exclusives les unes des autres, caractérisent au premier chef des situations nationales. La première résume les difficultés d'un marché $\mathrm{du}$ travail très fortement segmenté entre des «insiders» relativement peu nombreux, bénéficiant de fortes protections, et des «outsiders» manquant de sécurité comme de perspectives d'intégration dans le segment stable. On peut y voir une image stylisée des pays de type méditerranéen. La seconde se centre sur le manque de dynamisme global de marchés structurés autour des «marchés internes» traditionnels : les «insiders » sont ici plus nombreux et bien protégés, mais manquent de possibilités de rebond en cas de perte d'emploi. Ce sont alors les pays «continentaux» qui sont visés. Une troisième configuration met en avant des marchés du travail dynamiques mais souffrant d'un déficit de compétences et d'opportunités de promotions, risquant de bloquer les travailleurs les moins qualifiés dans des carrières à faible productivité. Ces dangers renvoient en particulier aux marchés du travail des pays de culture anglo-saxonne libérale. Enfin, la quatrième insiste sur les difficultés qui résultent d'un accès insuffisant au marché du travail formel, qu'elles soient dues à la prégnance du travail informel ou à la dépendance à l'égard des dispositifs de protection sociale et d'assistance. Ces difficultés caractérisent schématiquement les nouveaux pays membres issus de l'ancien bloc socialiste, mais aussi le défi de l'insertion des plus pauvres dans les pays de l'ancienne Europe.

Le rapport présente une série de préconisations en lien avec chacun de ces grands défis, préconisations qui mobilisent à des degrés divers les outils passés en revue plus haut. Elles dosent selon des proportions variables quatre composantes. L'une porte sur les arrangements contractuels, la seconde sur les politiques actives de l'emploi, la troisième sur la formation tout au long de la vie et la quatrième sur la protection sociale. On retrouve ici les trois pôles du schéma initial triangulaire, complétés par un pôle formation continue(4). Un examen plus détaillé permet de retrouver aisément les autres dimensions identifiées dans le quadrilatère que nous avons proposé plus haut, notamment les ajustements internes aux firmes et partenariaux, et la gestion territoriale et non marchande des transitions. Le triangle initial restrictif n'est mobilisé en tant que tel que dans une partie de la quatrième configuration, celle de la dépendance d'une population assistée.

S'il est clair que ce rapport met en avant la diversité européenne et réintègre assez largement les

(4) Une cinquième composante porte sur la confiance et le dialogue social, et a une portée procédurale. 
variables exclues dans la représentation tronquée que nous avons considérée initialement, on peut toutefois se demander si ces réponses n'en restent pas pour l'essentiel à un recueil de «bonnes pratiques», certes contextualisées, mais dont les conditions de fonctionnement conjoint ne sont pas examinées.

Il est donc intéressant de se pencher sur des versions plus ambitieuses, prenant appui plus explicitement sur le noyau central des «marchés transitionnels du travail». Deux idées passent alors au premier plan. La première, positive, est que les transitions font système, au sens où elles définissent un ensemble de places et d'opportunités qui conditionnent les stratégies et les anticipations des acteurs. Ce système est bien sûr ouvert, notamment en ce qui concerne les liens et les contraintes de la macroéconomie. La seconde, normative, est que ce sont les «transitions » qui doivent «payer» et non seulement ou non exclusivement le travail. La sécurité entretient des liens intimes avec la prise de risques dans un monde qui est soumis au changement rapide, et ne peut donc être séparée du traitement de l'innovation économique et sociale, et des prises de risques qui lui sont associées. Il doit donc être possible de conduire des carrières à plusieurs séquences et plusieurs orientations.

Alors l'insistance porte d'abord sur le contenu et l'évolution du socle minimal de droits et noyaux de stabilité qui sont nécessaires pour éviter les «dilemmes du prisonnier», dans lesquels personne n'est incité à prendre un risque, notamment en laissant un emploi jugé insatisfaisant. On trouve ici d'emblée un rôle clé et global pour l'Union européenne, d'abord dans la construction et la mise en place négociée de standards «transitionnels» qui évitent d'enclencher la course vers le bas. Droit à la formation, aux congés parentaux, à la réorientation... ces droits s'affirment certes sur une base nationale, mais commencent à trouver une homogénéisation communautaire, et ce mouvement doit être conforté. Mais ce portefeuille de droits nouveaux ne pourra s'enrichir que lentement compte tenu de la diversité des ressources et des avancées nationales. À un niveau sectoriel, d'autres standards devront toutefois se développer, tels que ceux qui régulent le fonctionnement des agences d'intérim, toujours dans le but d'éviter des spirales descendantes. Au niveau macroéconomique, l'établissement du socle se heurte à une difficulté évidente en ce qui concerne les salaires, dont les niveaux minima restent trop différents pour faire l'objet d'une harmonisation. La question peut alors se déplacer vers des standards minimaux en matière d'éducation.

Il est alors intéressant d'opérer une jonction avec les analyses formulées en termes de " variété du capitalisme» (Hall, Soskice, 2001 ; Amable, 2005). Leur principe central consiste en effet à mettre en avant et à exploiter les "complémentarités institutionnelles» entre régimes d'innovation, de financement et d'écoulement des produits d'une part, et régimes de formation et d'usage de la main-d'œuvre, d'autre part. Cette perspective principalement macroéconomique vient fournir une base analytique élargie aux combinatoires de la «matrice Schmid», insistant comme elle sur les jeux conjoints et les synergies. Une de ses principales conséquences en termes de politique économique (AMABLE, 2005; AMABLE, GATTI, 2006) est de montrer que les préconisations en termes de libéralisations, notamment celles qui pourraient affecter conjointement les marchés des produits et du travail, comportent souvent d'importants risques d'inefficacité et d'instabilité. Trois séries de complémentarités apparaissent pertinentes ici, complémentarités dont l'analyse pourrait faire l'objet de fertilisations croisées entre l'approche des «marchés transitionnels» et celle de la «variété du capitalisme». La première, évidente, est l'encastrement de la flexicurité dans les stratégies de stabilisation macroéconomique, notamment de contrôle et de sécurisation de la demande. C'est alors la question de la stabilisation contracyclique des revenus qui passe au premier plan, qu'il s'agisse de neutraliser les effets d'une contraction ou au contraire d'épargner des excédents temporaires. Les usages «transitionnels» d'outils tels que les comptes épargne-temps et comptes épargne salariale trouvent alors une justification supplémentaire. La seconde complémentarité vient s'intéresser à l'offre macroéconomique et aux interactions entre formation et innovation. Par exemple, dans les pays «continentaux » ayant développé des régimes d'innovation et de financement relativement centralisés et relativement protégés des marchés financiers internationalisés, de meilleurs ajustements du travail et de l'emploi supposent d'organiser une forte transférabilité des qualifications et l'aménagement concerté, souvent au niveau local, des mobilités. Dans d'autres configurations, le recours plus direct aux dynamiques et aux évaluations du marché peut en partie remplacer ce type de sécurité, mais la question des groupes cantonnés aux emplois de faible qualité reste posée. C'est alors un effort massif de formation en entreprise qui apparait prioritaire. Enfin, troisième ensemble de complémentarités, loin d'être inévitablement cantonné au marché du travail, le terme de flexicurité apparaît pertinent sur le marché des produits. Pour prendre un exemple central, une assurance efficace sur le prix de l'immobilier pourrait remplir des fonctions stabilisatrices et favoriser la prise de risque professionnel par les ménages les plus fragiles, tels que les jeunes qui se heurtent souvent aux défis d'obtenir un logement proche de leur emploi. On fait ainsi apparaître, et on exploite, une complémentarité entre les «transitions» et les marchés des biens et services. D'autres apparaissent avec l'ensemble des services aux personnes, du soin des enfants et des personnes âgées aux services de santé.

Quelle que soit la version retenue, ces préconisations ne peuvent éviter une interrogation sur la base 
des accords politiques susceptibles de générer de tels développements, et c'est là un second domaine dans lequel les liens entre MTT et «variété du capitalisme» sont en position d'apport réciproque. La mise en œuvre de la «flexicurité» suppose en effet la constitution de coalitions qui peuvent varier, tant dans leur composition que dans leur orientation. Elle soulève bien des réticences, et apparaît comme un compromis des élites portant pour la base relativement peu de bénéfices immédiats et davantage à long terme. On voit bien par exemple, et c'est l'apport des MTT, que la promotion d'accords locaux instaurant une mobilité protégée est une issue logique aux difficultés rencontrées par les pays de type «continental» ayant connu de forts «marchés internes». Toutefois, leur faisabilité concrète dépend non seulement d'innovations institutionnelles telles que les groupements d'employeurs, des négociations sur les «mobilités», mais encore de l'implication forte des exécutifs régionaux appuyés par des transferts décidés au niveau national, et donc, de la création d'une base politique favorable à ces perspectives. L'interrogation en termes de "variété du capitalisme», plus globale et plus pessimiste, se focalise précisément sur les conditions, difficiles à réunir, de constitution et de stabilité des coalitions électorales capables de porter des projets de ce type.

Face à une telle incertitude, on peut penser qu'au niveau européen, un processus politique doit renforcer le rôle permissif de l'Union et imposer son rôle catalyseur (SCHMID, 2008). On l'a vu, l'instauration de standards minima vient directement contrer les risques de cercles vicieux, et les pratiques de négociation collective trouvent dans ce domaine un point d'application essentiel. Mais l'Europe peut et doit aller au-delà. Dans le contexte actuel de subsidiarité et de faible développement de l'Europe budgétaire, une voie de sortie pourrait être une nette contribution à l'élévation du niveau de qualification des moins formés, par exemple sous la forme d'un big push visant à la réhomogénéisation de la force de travail européenne. Comme il a été analysé, une telle politique n'a de chance d'être efficace que si elle s'accompagne de l'organisation de «transitions», collectivement négociées et crédibles, pour ces mêmes travailleurs. Autrement, l'apparition d'accords partiels risque en permanence de rencontrer sinon l'hostilité, du moins la défiance des principaux acteurs concernés, et ainsi de manquer leur but de libération des initiatives.

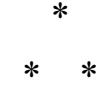

Il est à peine besoin, au terme de cette esquisse, d'en souligner les limites. Son ambition normative s'appuie sur de nombreux travaux positifs qui n'ont pas été présentés ici, et les quelques illustrations fournies ne peuvent tenir lieu d'applications. La perspective des MTT, si ambitieuse qu'elle puisse paraître, demeure partielle et suppose par exemple une articulation à expliciter avec la macroéconomie et avec les processus d'innovation. Nous espérons toutefois avoir montré comment les objectifs et processus souvent confus voire contradictoires associés au terme de «flexicurité» pouvaient être clarifiés au moins partiellement par la référence aux MTT. Ceux-ci conduisent à rejeter l'interprétation réduite à une baisse de la protection de l'emploi, pour mettre en évidence et préciser le rôle joué par les partenaires sociaux, les firmes et les territoires dans l'aménagement de mobilités protégées et de noyaux de stabilité.

\section{Bibliographie}

Amable B., (2005), Les cinq capitalismes, Éditions du Seuil.

Amable B., Gatti D., (2006), «Labor and product market reforms : questionning policy complementarity», Industrial and Corporate Change, vol. 15, $\mathrm{n}^{\circ} 1, \mathrm{p} .101-$ 122

Anxo D., Erhel C., (2006), «Irreversibility of time, reversibility of choices? The life-course foundations of the Transitional Labour Market Approach», Cahiers de la MSE, 2006.58.

Auer P., SCHMid G., (1997), «Transitional Labour Markets. Concepts and Examples in Europe», in European Academy of the Urban Environment, New Institutional Arrangements in the Labour Market, Berlin.
Auer P., Cazes S. (dir.), (2003), Employment stability in an age of flexibility, Genève, BIT.

Auer P., Gazier B., (2006), L'introuvable sécurité de l'emploi, Flammarion.

BARBier J.-C., (2006), «Au-delà de la "Flex-sécurité", une cohérence sociétale solidaire au Danemark», in Paugam S. (dir.), Repenser la solidarité, Paris, Presses universitaires de France, p. 473 - 490.

BOyer R., (2006), La flexicurité danoise. Quels enseignements pour la France?, Éditions Rue d'Ulm/Presses de l'École normale supérieure, collection du CEPREMAP, avril.

Bredgaard T., Larsen F., Madsen P.K., (2006), «Opportunities and challenges for flexicurity - The 
Danish example», Transfer, European Review of Labour and Research, vol. 12, $\mathrm{n}^{\mathrm{o}}(1), \mathrm{p} .61-80$.

Cahuc P., Kramarz F., (2005), De la précarité à la mobilité. Vers une sécurité sociale professionnelle, La Documentation française.

Cazes S., Nesporova A., (2007), Flexicurity. A relevant approach in Central and Eastern Europe, BIT.

Commission européEnNe, (2006), Employment in Europe, Bruxelles.

De Koning J., (2002), «Transitional Labour Markets : an economist's view », in Mosley H., O'Reilly J., Schömann K. (dir.), Labour Markets, Gender and Institutional Change. Essays in Honour of Günther Schmid, Edward Elgar, p. $290-315$.

ERHEL C., (2007), «Les politiques liées au cycle de vie et le marché du travail», Séminaire Delsa/Elsa sur les risques de l'existence, cycle de vie et politique sociale, OCDE, 31 mai et $1^{\text {er }}$ juin.

EUROPEAN FOUNDATION FOR THE IMPROVEMENT OF LIVING CONDITIONS (2007), Varieties of flexicurity: reflections on key elements of flexibility and security, Hearing on the Commission's green paper «Modernising labour law to meet the challenges of the 21st century», European Parliament Committee on Employment and Social Affairs, 21 March.

EYMARD-Duvernay F., (2006), «Introduction», in Eymard-Duvernay F. (dir.), L'économie des conventions. Méthodes et résultats, La Découverte, Tome 1, p. 11 -20 .

FONDS MONÉTAIRE INTERNATIONAL, (2003), «Unemployment and Labor Market Institutions: why Reforms Pay Off», World Economic Outlook, ch. 4.

Gazier B., (2000), «L'articulation justice locale/justice globale. Le cas des "marchés transitionnels du travail"»", Revue Économique, mai, p. 571-581.

GAZIER B., (2002), «Transitional Labour Markets : from positive analysis to policy proposals», in Schmid G., Gazier B. (dir.), The Dynamics of Full Employment. Social Integration Through Transitional Labour Markets, Edward Elgar.

GAzier B., (2005), Vers un nouveau modèle social, Flammarion.

GAZIER B., (2007), «Restructurations et reclassements : vers une redéfinition des responsabilités », Droit Social, $\mathrm{n}^{\mathrm{o}} 3$, mars, p. 259-267.

Gazier B., Lechevalier A., (2006), «Stratégie européenne de l'emploi, régimes macroéconomiques et institution- nels, et marchés transitionnels du travail», in Dang A.T., Outin J.-L. et Zajdela H. (dir.), Travailler pour être intégré? Mutations des relations emploi - protection sociale, CNRS Éditions, p. 218 - 234.

Hall P.A., Soskice D. (dir.), (2001), Varieties of Capitalism : the Institutional Foundations of Comparative Advantage, Oxford U. Press.

JEPSEN M., (2005), «Towards a gender impact analysis of Flexicurity», in Bredgaard T., Larsen F. (dir.), (2005), Employment Policy from Different Angles, Copenhagen, DJØF Publishing.

Leschke J., Schmid G., Griga D., (2006), «On the marriage of flexibility and security: lessons from the Hartz reforms in Germany », WZB Discussion Paper, SPI 2006 - 108, avril.

OCDE, (2006), Perspectives de l'emploi, OCDE.

Ramaux C., (2006), Emploi: éloge de la stabilité. L'État social contre la flexicurité, Mille et une nuits.

Schmid G., (1995a), «Is Full Employment Still Possible? Transitional Labour Markets as a New Strategy of Labour Market Policy», Economic and Industrial Democracy, vol. 16 (3), p. 429-456.

SCHMid G., (1995b), «Le plein-emploi est-il encore possible? Les marchés du travail "transitoire" en tant que nouvelle stratégie des politiques d'emploi», Travail et Emploi, no 65, p. 5-17.

Schmid G., (2006), «Social Risk Management through Transitional Labour Markets», Socio-Economic Review, $4(1), 1-33$.

Schmid G., (2008), Full Employment in Europe. Managing Labour Market Transitions and Risks, Edward Elgar, à paraître.

Schmid G., GAzIer B. (dir.), (2002), The dynamics of full employment. Social integration through Transitional Labour Markets, Edward Elgar.

Supiot A., (1999), Au-delà de l'emploi, Flammarion.

Wilthagen T., Tros F., (2004), «The concept of "Flexicurity": a new approach to regulating employment and labour markets », Transfer, 10 (2), p. 166-186.

Wilthagen T., (2005), «Striking a Balance? Flexibility and Security in European Labour Markets », in Bredgaard T, Larsen F. (dir.), Employment policy from different angles, DJØF Publishing Copenhagen, p. 253-267.

Wilthagen T. (rapporteur), (2007), Flexicurity Pathways. Turning Hurdles into Stepping Stones, Report by the European Expert Group on Flexicurity, Bruxelles, juin, $41 \mathrm{p}$. 